\title{
Strategic paths and memory map: Exploring a building and memorizing knowledge
}

\author{
Sandro Varano ${ }^{1}$, Jean-Claude Bignon ${ }^{2}$ \\ MAP CRAI UMR nº64/CNRS/CULTURE \\ ${ }^{1}$ Ecole Nationale Supérieure d'Architecture de Strasbourg, 8 Boulevard du Président \\ Wilson, 67000 Strasbourg, France \\ ${ }^{2}$ Ecole Nationale Supérieure d'Architecture de Nancy, 2 rue Bastien Lepage, BP 40435, \\ 54001 Nancy, France \\ \{varano, bignon\}@crai.archi.fr
}

\begin{abstract}
Restoring archaeology and architecture, we propose a 3D navigation mode based on topographic and cognitive paths. During the exploration of a 3D model, the learner can create his own memory map facilitating the appropriation and memorization of knowledge. In this article, we will correlate the exploration and creation activities. The Great Pyramid of Giza is a support to this work.
\end{abstract}

Keywords: Archaeology and architecture, strategic path, memory map, learning.

\section{Introduction}

This work deals with archaeological and architectural restoration. The aim is to teach the public by facilitating the appropriation and memorization of new knowledge of cultural heritage. The system presented proposes a 3D navigation mode based on strategic paths dedicated to learning.

In our system, the real-time visit of an archeological site or an architectural monument leads to two activities that the learner performs in parallel:

- the exploration of the 3D model

- the creation of the memory map

These activities belong to a real educational project: the exploration based on clues discovery and riddles resolution incites the learner to participate; the creation helps the learner to organize and visualize information. Both processes allow him to structure and construct knowledge.

During the exploration activity, the learner is guided and motivated in sequenced and superimposed routes, while allowing a lot of freedom. This structure in double layers is composed of the topographical path and the cognitive path. During the creation activity, the learner materializes his mental map. The memory map evolves according to the progress of the learner on these paths. 
In this research work, we will correlate the creation of the memory map with the kind of narrative proposed during the exploration. In our study, we will choose the Great Pyramid of Giza in Egypt. With the help of Tristan Truchot, we created a prototype of an extract of the scenario to estimate and experiment our work.

\section{Strategic paths}

\subsection{The topographical path}

To be able to structure the movements, we introduce the notion of the topographical path by identifying critical points and secondary points in the path and by putting them in concordance. It is necessary to choose in the studied building the interesting critical points according to the message that we want to communicate to the learner. Each crossing point suggests specific actions that we wish to represent in the topographical path. The crossing points of the path consist of two types of points:

- the information points, defining the information route

- the knowledge points, defining the knowledge route

The data on the information route are reinvested in the knowledge points where the learner transforms the information into knowledge. The knowledge point is a critical point structuring the path in sequences.

The successive sequences define a quest. The number of information points in each sequence is defined in relation to the riddle proposed and the number of knowledge points in a quest is defined by the message (religious, structural aspect, etc.) to be taught (fig. 1).

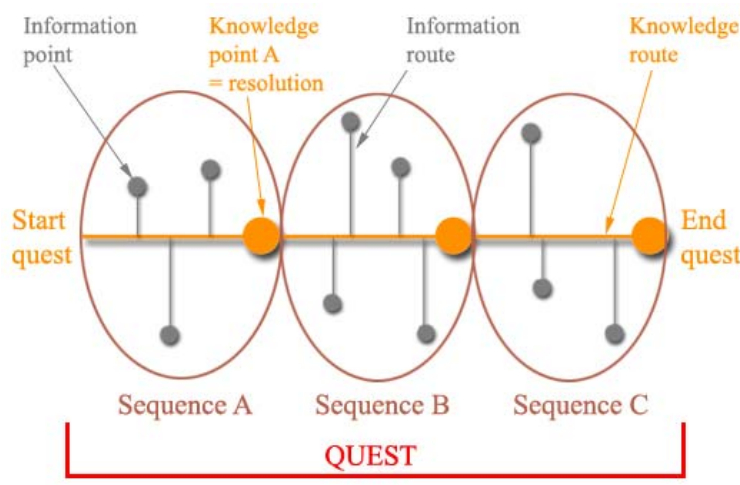

Fig. 1. Successive sequences of the path defining a quest. 


\subsection{The cognitive path}

Research and cognitive experiments have shown that the learner must be constantly alerted and motivated to be interested in the studied topic. The acquisition of new knowledge depends on his willingness to achieve the objectives defined at the beginning.

To motivate the learner by avoiding a "cognitive overload" [1], we define a ludic aspect of the path in relation to the diversity of spaces that can be visited and in relation to the possible interactions in these spaces by manipulating multimodal information.

We decide to create some extensions of the path in the 3D base model. These spaces voluntarily added, fictitious or depending on the historical context of the building, belong to the information route and hold the clues for solving the riddle at the knowledge point and for moving to the next sequence. The knowledge points would thus be existing spaces in a building to be communicated. The transition between the existing spaces and added spaces is possible by crossing portals.

Imagine a path in the Cheops Pyramid in Egypt. The learner moves in real time in the existing spaces in the monument. He can take at any time inserted exterior spaces. By leaving the "King's Chamber", he could go for example to a mummification room of the second empire, to the Louvre Museum in Paris, to an invented virtual space allowing an interaction between the learner and his environment.

\section{The memory map}

During the creation activity, the learner outlines his path. Like a cartographer, he reveals his discoveries in order to establish a journal telling the story of his journey.

\subsection{A multimedia notebook}

According to David Cohen, multimedia has three components: Audiovisual, Interactivity and Network [2].

The memory map is a support crossing various graphic and sound representations: it allows the learner to store images, take notes, sketch drawings and play audio or video: multimodal information found during the 3D exploration or created by the learner. Information contained on the map is manipulated. Actions such as moving, connecting, correcting, removing, etc, maintain an interaction between information and the user.

The memory map is not a closed system with its own data library. Connections are possible with Internet. This concept of network extends the notions of space and distance between consulted information and the learner. 


\subsection{A locating tool}

Outlining a path to explain where we have come from but also where we are going. In this context, a rapid movement system is thus established to navigate between the memory map and the 3D model. This feedback system is possible using sensitive areas. Teleportation provides a transfer between two points of view: an internal or subjective focus on the building and a zero focus around the memory map.

\subsection{A memory tool}

The memory map transmits messages. Its capacity to produce meanings allows the learner to build his own reasoning. According to Jean Piaget [3], knowledge results from the interaction between the person and the environment, compared to the constructivist hypothesis: we appropriate knowledge that we build ourselves.

The possible links between the information allow the learner to create associations between elements. This process is similar to the mnemonic method. This activity is a learning process by organizing information and by encouraging memorization.

\section{A scenario in the Cheops Pyramid}

A prototype is realized using the Java language in order to create the memory map and the level editor Unreal Ed 4.0 (with the video game Unreal Tournament 3 [4]) in order to create a 3D model of the pyramid (fig.2). The method consists in using the Internet protocols (TCP and HTTP) to put in relation the 3D model and the memory map.

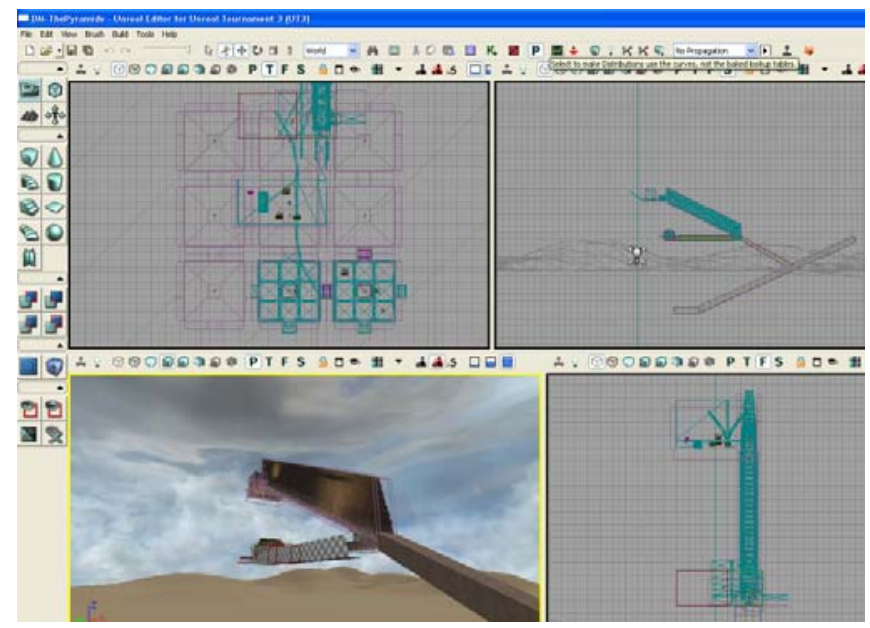

Fig. 2. The Cheops Pyramid in the level editor Unreal Ed 4.0. 
The Great Pyramid contains many mysteries. Its architectural, symbolic or historic complexity, allows us to define several quests. In our study, we approach the religion of the pyramid.

\subsection{Story of a journey into the afterlife}

The pyramid is the funeral monument allowing the pharaoh to live eternally. The purpose of the quest is to reveal the journey of the pharaoh to reach the afterlife.

The learner will discover through the pyramid a considerable universe of symbols and religious practices assuring the rebirth of the deceased.

He will move in the monument with a subjective point of view embodying the soul of the pharaoh Cheops.

The quest possesses four riddles or four knowledge points (fig. 3):

- the entrance: the cosmos perceived from the Nile

- the Great Gallery

- the "Queen's Chamber": a path of obstacles

- the "King's Chamber”: return to his sarcophagus without mistakes

Each solved riddle delivers a canopic jar to the learner. The four canopic jars collected allow him to reach the objective of the quest.

As an example, we will develop the knowledge point $\mathrm{C}$.

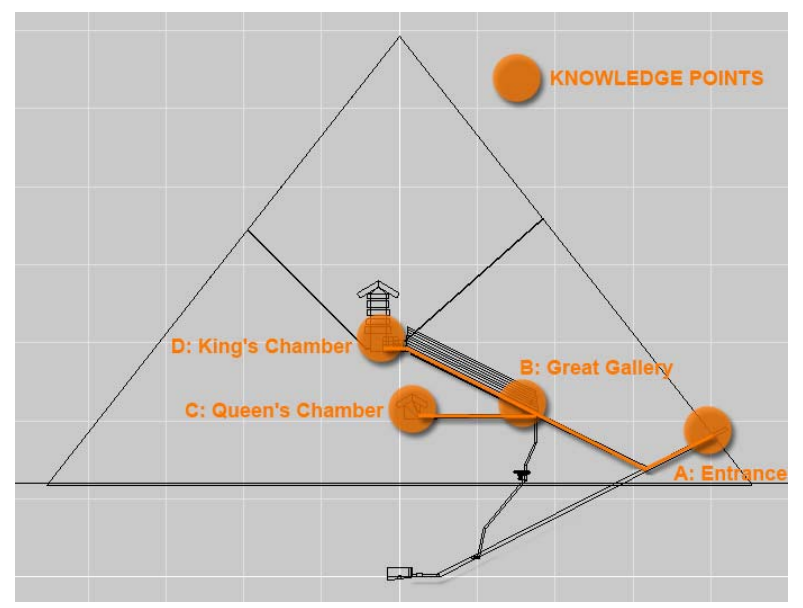

Fig. 3. The route of knowledge in the Cheops Pyramid.

\subsection{The riddle of the “Queen's Chamber”}

The “Queen's Chamber” has its name by mistake. In fact, the King's wives had their tombs near the Great Pyramid. This chamber, and in particular the North and South 
ducts, question researchers. These ducts are blocked by limestone doors possessing two copper handles.

The riddle is to discover the ducts and their astronomical and religious functions.

\subsection{The information spaces}

The number of information spaces results from the riddle of the chamber. We report three spaces and we will develop one to study the possible actions:

- Reading space: the invisible and immaterial entities of human being

- Immersion space: a journey by solar bark

- Logic and mechanism space: the weighing of the heart

This space informs about the ritual obstacles that the pharaoh must pass for his resurrection in the afterlife, materialized by the doors blocking the ducts.

In reference to the film Cube [5], the logic and mechanism space is constituted by attached boxes in three dimensions (fig. 4). These rooms and the passages assuring their communication are a labyrinth. They are a metaphor of traps and doors that the pharaoh must face.

The deceased must prove his purity by finding and reciting magic words. In the judgment room possessing scales, his weighed heart is then lighter than the feather of Maât.
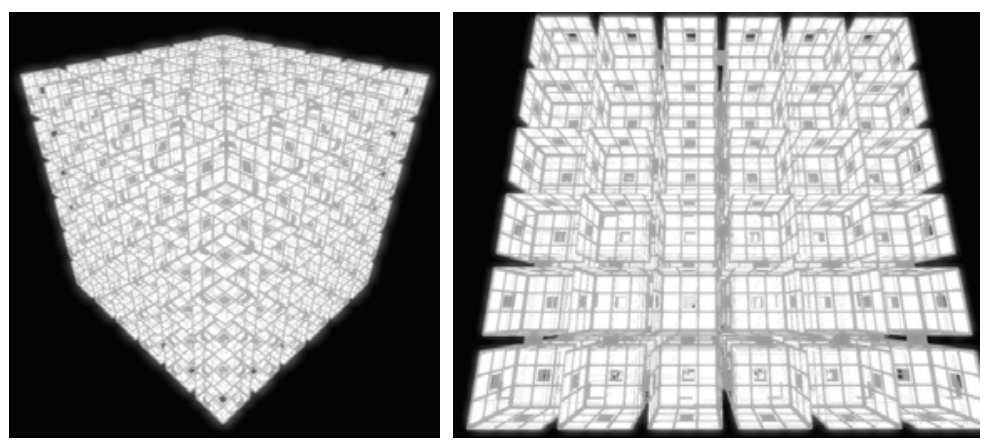

Fig. 4. The logic and mechanism space based on the film Cube.

The events are the following:

- The portal located in the horizontal corridor of the pyramid (fig. 5) teleports the learner to the first room. This room possesses an extract of the Egyptian Book of the Dead (Papyrus of Hunefer, British Museum, London) illustrating the weighing of the heart and the judgment. 


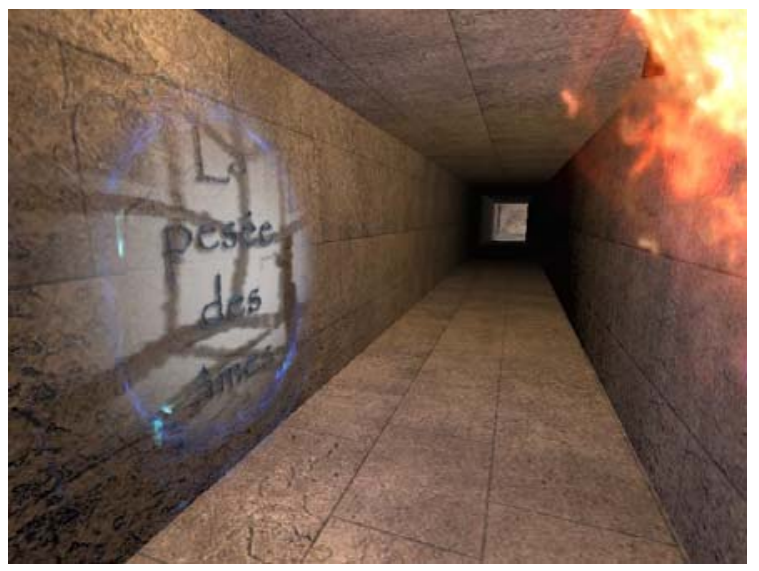

Fig. 5. Teleportation portal located in the horizontal corridor.

- Each room possesses six doors with symbols based on the Book of the dead. The doors are present to guide the learner (fig. 6). There are the doors guarded by the hostile creatures Ammout and Apophis (crocodile, snake, insect, etc) and by passing through them, the learner is blocked in a room and is forced to come back.

- There are also the doors indicating the route of confession (feather, jar, etc). By passing through them, the learner discovers a fragment of papyrus mentioning a magic word to be recited (“I didn’t incite to cry”, “I didn’t ordain to kill”, etc).

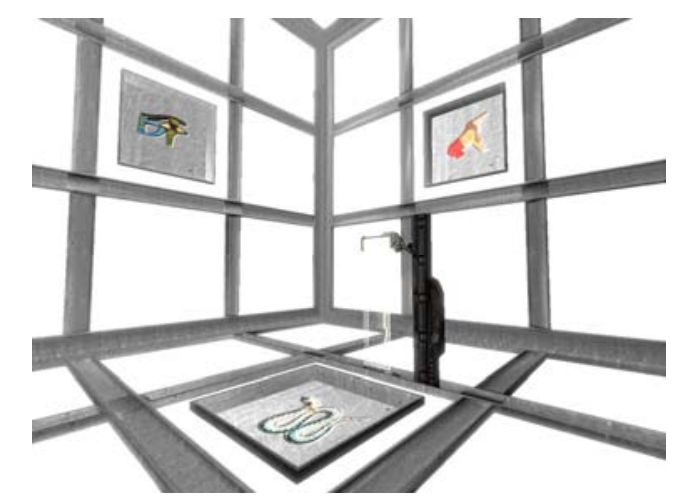

Fig. 6. A room and its doors in the logic and mechanism space.

- The route of confession leads to the judgment room. It possesses scales in its center, a feather and a jar. Forty two judges and five gods Anubis, Maât, Thot, Horus and Osiris (frescoes on walls) attend the weighing done by the learner.

- A portal allows the learner to return into the pyramid. Then, he explores the other information spaces or goes to the "Queen's Chamber" to resolve the riddle. 


\section{Correlation between the memory map and the strategic path}

Each knowledge point allows the realization of a screenshot. It is a page of the memory map as a support of representation. When the learner visits the knowledge point, he initializes automatically the screenshot illustrating the space where he is (fig. 7). Thus, the number of knowledge point determines the number of pages of the memory map.

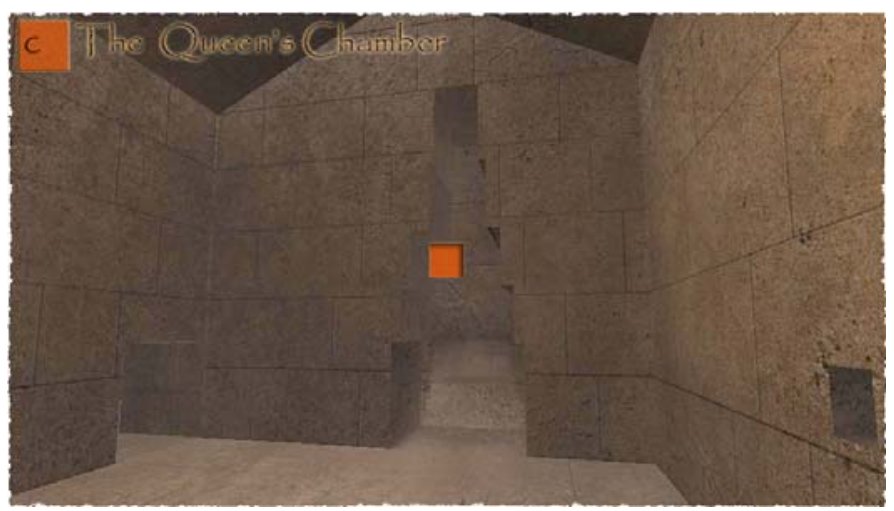

Fig. 7. A screenshot in the « Queen’s Chamber».

Then, the learner builds his relation to the spaces using words and images related with the information spaces: he clicks on the information to display on a page; he writes key sentences; he draws details or plans.

In the information space Cube, the learner first stores the papyrus discovered in the first room. Thereafter, he can relate the symbols perceived on the doors to those of the papyrus, he then sketches the plan of the labyrinth to find his way and he writes magic words to remember (Fig. 8).

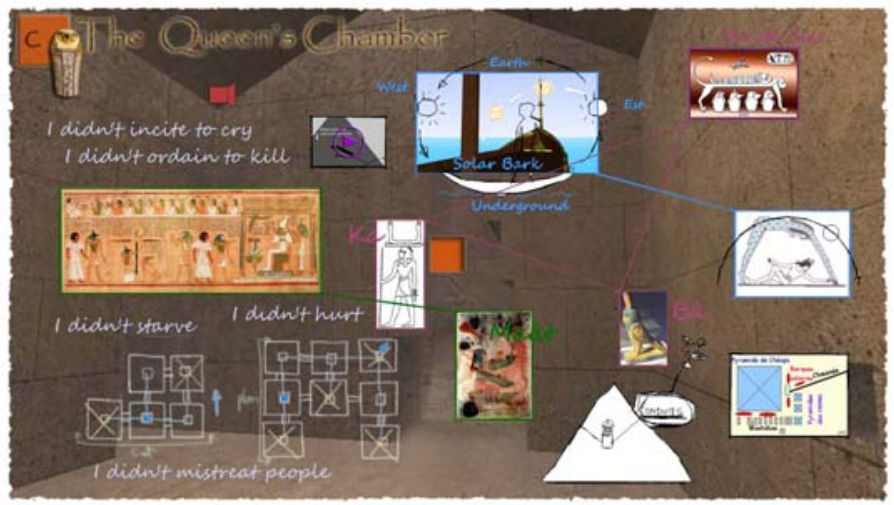

Fig. 8. A personalized screenshot.

Two types of links are managed in the system: 
- The automatic links connect the information of the same space (2D links on a page) and the various knowledge points (3D links between pages).

- The personalized links created by the learner to connect similar elements.

When the riddle is solved, a canopic jar appears on the page.

The learner then begins the following sequence. He discovers the new riddle in the knowledge space and generates a new page of the memory map. The pages are added until the end of the quest (fig. 9).

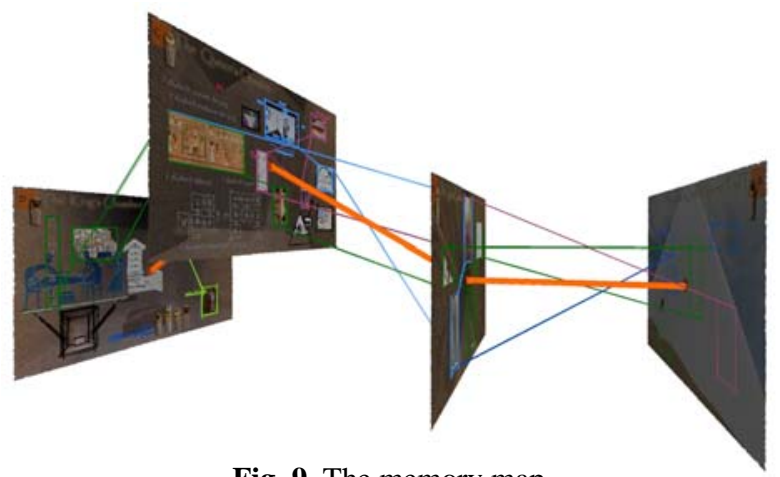

Fig. 9. The memory map.

A teleportation script (fig. 10) manages the transfers between the pages and knowledge spaces of the 3D model by clicking sensitive areas.

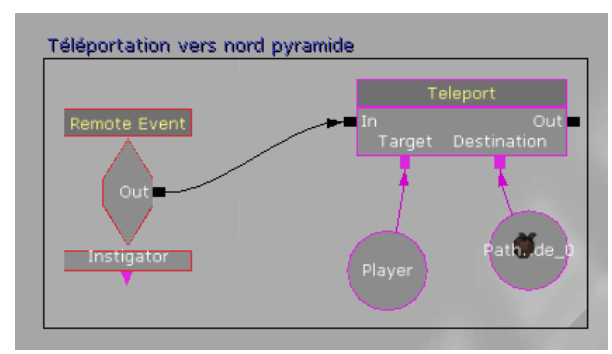

Fig. 10. A teleportation script in the level editor Unreal Ed 4.0.

\section{Conclusion}

During the visit of a 3D model, the exploration and creation activities allow us to elaborate a learning system supervising the navigation of the learner: by managing his movements and by taking into account his cognitive capacities.

We have seen how the creation of the memory map is based on the structure of the topographic and cognitive paths. The map accompanies the learner's exploration. 
On the one hand, the paths manage movements and possible interactions in the spaces; on the other hand, the memory map retains traces as evidence of an exploration.

By producing meaning and by evoking his limits, the learner tries to master the unknown by his own representation.

Finally, the realization of the prototype shows some technical or screenplay problems. This prototype will allow us to validate or revise our work. An experiment is planned with students.

\section{References}

1. Jacquinot, G.: Les NTIC : écrans du savoir ou écrans au savoir. In: Chevalier, Y., Ateliers 9/1996, Outils multimédias et stratégies d'apprentissage du FLE, Cahiers de la Maison de la recherche, tome 1, Université Charles-de-Gaulle Lille 3, Lille (1996)

2. Cohen, D.: Interfactives ou l'écran agi. Les métaphores à l'écran. In: Écrits. Images. Oral et Nouvelles technologies. Actes du séminaire 1994-1995. Under the responsability of MarieClaude Vettraino-Soulard, Université Paris 7-Denis Diderot (1995)

3. Piaget, J.: La naissance de l'intelligence de l'enfant. Delachaux \& Niestlé, Neuchâtel (1936)

4. Unreal Tournament 3, 2007, Epic Games, Midway Games: http://www.unrealtournament3.com/

5. Natali, V.: Cube. Metropolitan FilmExport (1999) 